\title{
ARTICLE OPEN Magnetism of graphene quantum dots
}

\author{
Yuanyuan Sun ${ }^{1,2}$, Yongping Zheng ${ }^{1}$, Hongzhe Pan ${ }^{1}$, Jie Chen ${ }^{1}$, Weili Zhang ${ }^{1}$, Lin Fu ${ }^{1}$, Kaiyu Zhang ${ }^{1}$, Nujiang Tang ${ }^{1}$ and Youwei Du ${ }^{1}$
}

Graphene quantum dots with the high edge-to-area ratio have possibly substantial spin polarized edge states, which theoretically can generate fascinating magnetic properties. The magnetism of well-defined graphene quantum dots is relevant with both fundamental physics and potential applications in spintronics. In this article, we report the intrinsic magnetism of graphene quantum dots. Our graphene quantum dots with the average diameter of ca. $2.04 \mathrm{~nm}$ show the purely Curie-like paramagnetism with the local moment of $1.2 \mu_{\mathrm{B}}$ at $2 \mathrm{~K}$. It is proposed that the magnetic moment of graphene quantum dots may mainly origin from the residual zigzag edges passivated by hydroxyl groups. The ratio of nonmagnetic graphene quantum dots is approximately $6 / 7$, with most of the magnetic edge states suppressed by edge defects and/or edge reconstruction arising from the high-temperature annealing. Our study experimentally unveils the intrinsic magnetism of graphene quantum dots.

npj Quantum Materials (2017)2:5 ; doi:10.1038/s41535-017-0010-2

\section{INTRODUCTION}

Graphene quantum dots (GQDs) with a tiny size of only several nanometers present extraordinary properties due to quantum confinement $^{1}$ and edge effects, ${ }^{2}$ which attract a great deal of interest lately in the fields of bio-imaging, sensors, catalysis, photovoltaic devices, supercapacitors, etc. ${ }^{3}$ Notably, the magnetism of GQDs, because of their spin polarized edge states and potential applications in the spintronic devices, ${ }^{4-8}$ has aroused continual and tremendous interest. Due to the high edge-to-area ratio and the possible substantial spin-polarized edge states at the zigzag segments, the magnetism of GQDs was theoretically predicted to be especially intriguing. ${ }^{9-13}$ Experimentally, the spin-polarized edge states on zigzag edges of single narrow graphene nanoribbon have shown room-temperature magnetic ordering, raising hopes of spintronic devices operating under ambient conditions. ${ }^{14}$ Up to now, researches on the edge statesinduced magnetism of GQDs are mainly by theoretical approaches. ${ }^{11,12}$ For example, theoretical researches predicted that the geometric shape plays an important role in the magnetic properties of GQD. ${ }^{9-11,15}$ In addition, both defects and reconstruction at the edge were reported to switch off the spin polarization of the edge states. ${ }^{16-18}$ Despite this, the spin polarized edge states have also been predicted to be robust against shape irregularity and edge roughness. ${ }^{15,19-23}$ Clearly, the theoretical researches on the edge states magnetism of GQDs with naturally complicated boundary are highly controversial. Therefore, to unveil the edge states magnetism in GQDs, experimental investigation on the magnetism of intrinsic GQDs is of great significance and highly anticipated. ${ }^{11,12}$

Unfortunately, the low yield and purity of GQDs all along, ${ }^{24}$ which is insufficient for magnetism detection by macro-magnetic measurement, limit experimental research on the magnetism of GQDs. Annealing graphene oxide (GO) powder in an argon atmosphere is a commonly established method for mass production of graphene powder. Our recent progress in the production of monolayer GO quantum dots (GOQDs) with high yield and purity provides an opportunity for the mass production of high-purity GQDs. ${ }^{25}$ In this work, high-purity GQDs with mass production were obtained by annealing monolayer GOQDs in argon atmosphere at $1010^{\circ} \mathrm{C}$. We report the intrinsic magnetism of GQDs (ca. $2.04 \mathrm{~nm}$ ) with mostly pristine edges and nearly perfect basal plane. The GQDs show the purely Curie-like paramagnetism at $2 \mathrm{~K}$. However, the majority of GQDs are nonmagnetic, which may attribute to defects and/or reconstruction at the edge arising from the high-temperature annealing. The magnetism of GQDs may mainly origin from the residual edge states passivated by hydroxyl groups.

\section{RESULTS}

Characterization of the GQDs sample

The synthetic scheme for GQDs is shown in Fig. 1. Monolayer GOQDs, as the precursor with high yield and purity, were produced by acid-assisted cleavage of carbon black as reported previously. ${ }^{25}$ And then, the GQDs sample was obtained by annealing GOQDs in argon atmosphere at $1010^{\circ} \mathrm{C}$ for $1 \mathrm{~h}$ (see Materials and Methods).

The morphologies of GQDs were investigated by transmission electron microscopy (TEM) and atomic force microscope (AFM). The typical TEM image of GQDs is shown in Fig. 2a, indicating that the size of GODs is relatively uniform. The upper left inset of Fig. $2 a$ is the high-resolution transmission electron microscopy (HRTEM) image with an in-plane (0-110) lattice spacing of ca. 0.23 $\mathrm{nm}$, revealing the high crystallinity of GODs. The diameter distribution of GQDs is shown in Fig. 2b, which ranges approximately over $1.44-3.01 \mathrm{~nm}$. The corresponding average diameter $D_{\mathrm{a}}$ is ca. $2.04 \mathrm{~nm}$, much smaller than that of the precursor GOQDs (ca. $4.13 \mathrm{~nm}$ ). ${ }^{25}$ Figure $2 \mathrm{c}$ shows the AFM image of GQDs. A statistically analysis on nearly 100 quantum dots gives the GQDs' topographic height of approximately $0.35-0.88 \mathrm{~nm}$ (Fig. 2d). The corresponding average height is ca. $0.52 \mathrm{~nm}$, implying that most of the GQDs are mono or bi-layer. Namely,

\footnotetext{
${ }^{1}$ National Laboratory of Solid State Microstructures, Collaborative Innovation Center of Advanced Microstructures, Jiangsu Provincial Key Laboratory for Nanotechnology, Nanjing University, Nanjing 210093, China and ${ }^{2}$ Institute of Condensed Matter Physics, School of Science, Linyi University, Linyi 276000, China

Correspondence: Nujiang Tang (tangnujiang@nju.edu.cn)
}

Received: 25 August 2016 Revised: 29 December 2016 Accepted: 10 January 2017

Published online: 30 January 2017 


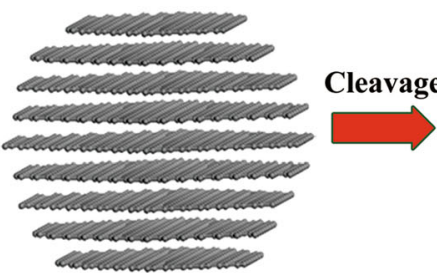

Carbon black
GOQD

GQD

Fig. 1 Synthetic scheme for GQDs. The black and red balls denote carbon and oxygen atoms, respectively

a

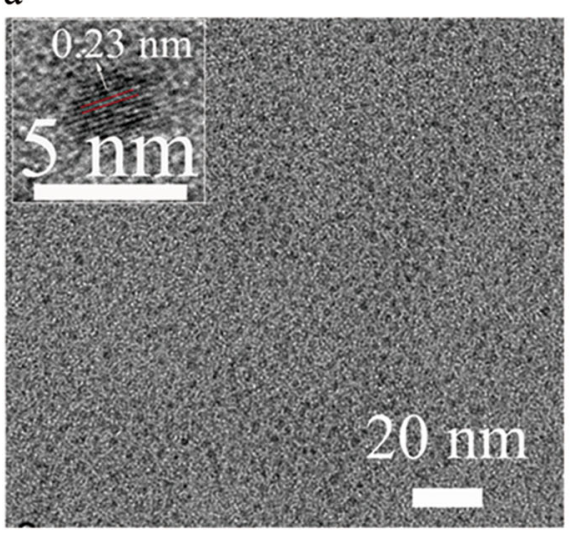

c

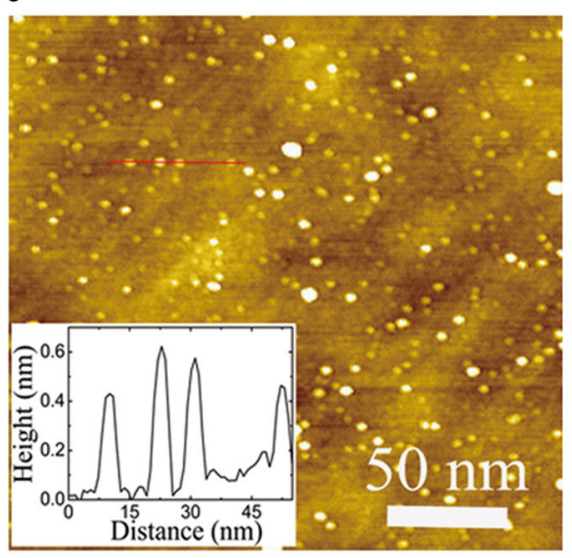

b

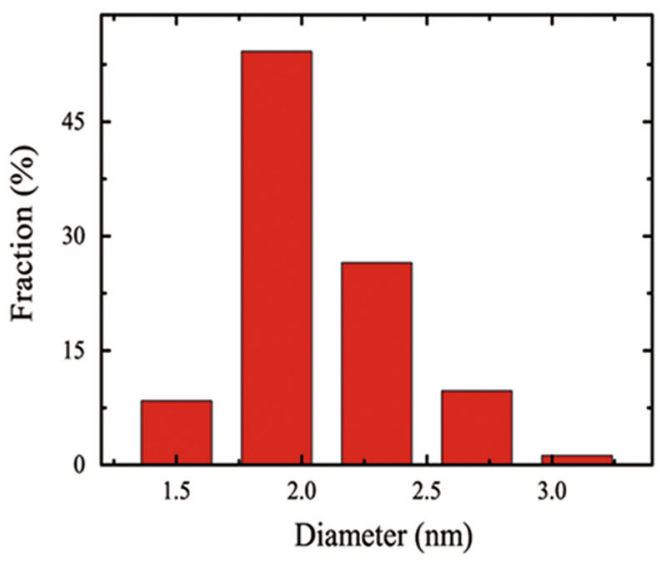

d

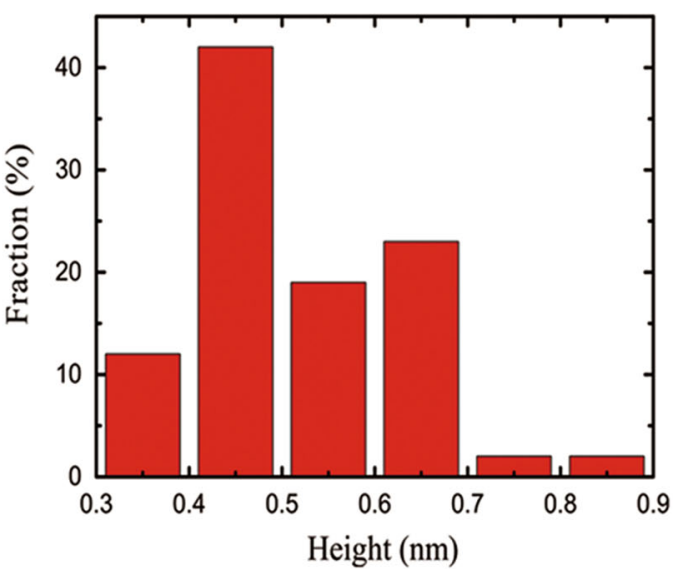

Fig. 2 a TEM image of GQDs. The upper left inset is HRTEM image. b The diameter distribution of GQDs. c AFM image of GQDs. The lower left inset is the height profile of the red line. $\mathbf{d}$ The height distribution of GQDs

the mono or bi-layer GQDs with narrow size distribution were obtained.

To examine the deoxygenation efficiency of GQDs obtained by annealing GOQDs at the high temperature of $1010^{\circ} \mathrm{C}$, X-ray photoemission spectroscopy (XPS) measurements were performed. Figure 3a shows the XPS spectra of GQDs and the original material carbon black for comparison. The residual oxygen content in GQDs (defined as O/C $\times 100$ at.\%) is $~ 5.73$ at.\%, and the oxygen content in carbon black is $\sim 5.74$ at.\%. The oxygen in carbon material was reported to be inevitable due to the atmospheric oxidation. ${ }^{26,27}$ Notably, the oxygen content of GQDs is similar to that of the original material carbon black, suggesting that the annealing at $1010^{\circ} \mathrm{C}$ is sufficient to eliminate most of the oxygen functional groups. Since the annealing temperature is as high as $1010^{\circ} \mathrm{C}$, the residual oxygen in GQDs can only bond at the edge. ${ }^{27-30}$ To make clear which kinds of residual oxygen functional groups these are, the typical fine-scanned $C$ 1s spectrum of GQDs was carefully deconvoluted into five peaks (Fig. 3b), which are respectively $\mathrm{C}-\mathrm{C} s p^{2}(284.6 \mathrm{eV}), \mathrm{C}-\mathrm{OH}$ (hydroxyl, 285.4 eV), C-O-C (ether, $286.2 \mathrm{eV}$ ), C=O (carbonyl, $287.7 \mathrm{eV})$ and $\pi-\pi^{*}(290 \mathrm{eV}) .^{25}$ It is clear that despite most of the edges of GQDs are pristine, a small amount of edges are bonded by hydroxyl, ether and carbonyl.

High-temperature annealing removed the oxygen functional groups on the basal plane, which may more or less generate vacancies on the basal plane of GQDs sheet. Since carbon atoms in the basal plane may rearrange during the high-temperature annealing due to the available thermal energy, ${ }^{31}$ vacancies in the basal plane of the small-size GQDs sheets may move to the edge and thus recover the $s p^{2}$ network structure. To identify the 
a

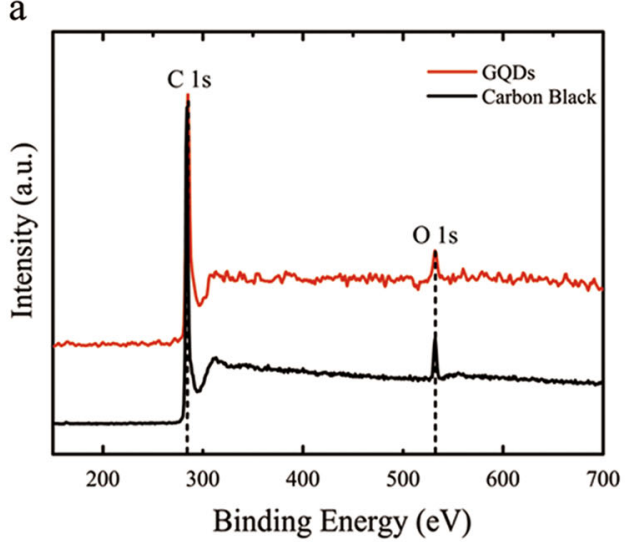

C

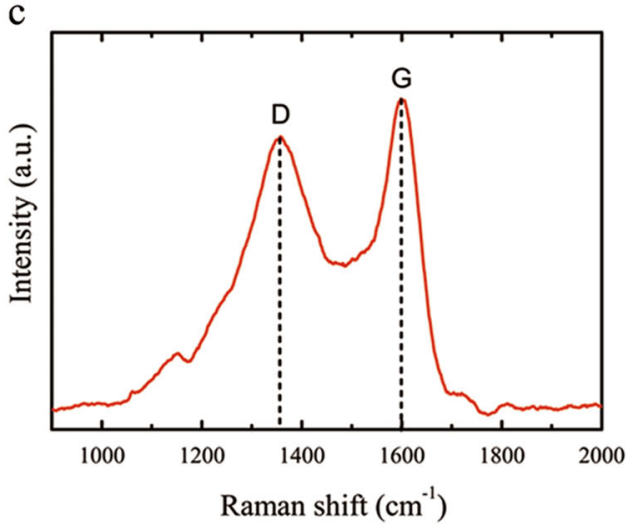

b

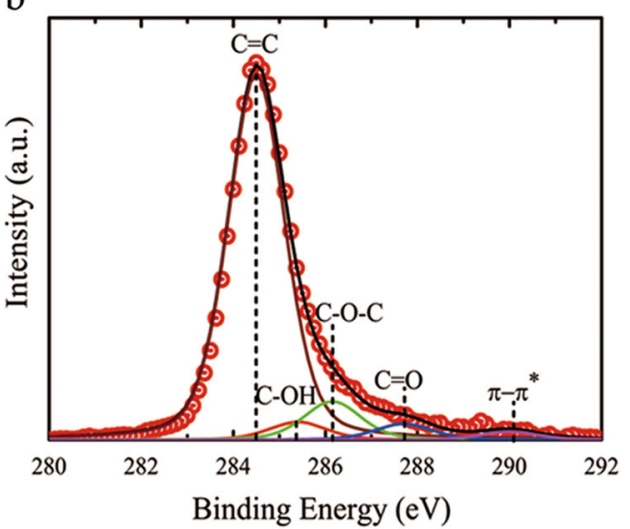

Fig. 3 a XPS spectra of GQDs and the original carbon black over a wide range of binding energies (150-700 eV). b Fine-scanned C $1 \mathrm{~s}$ XPS spectrum of GQDs. c Raman spectrum of GQDs

recovery of the $s p^{2}$ network structure of GQDs, Raman spectrum (Fig. 3c) was measured to detect the crystallite size. The $D$ peak is located at $1357 \mathrm{~cm}^{-1}$, while the $G$ peak is located $1598 \mathrm{~cm}^{-1}$. The crystallite size $L_{\mathrm{a}}$ can be obtained by the following equation

$L_{\mathrm{a}}=\frac{4.4}{R}\left(\frac{2.41}{E_{\mathrm{I}}}\right)^{4}$,

which is adapted from the Tuinstra-Koenig law to include the energy dependence; ${ }^{32-35} R$ is the integrated intensity ratio $I_{D} / l_{\mathrm{G}}$, and $E_{1}$ is the excitation laser energy. The fitted value of $R$ is 2 , while the laser line wave length used in the measurement is $514 \mathrm{~nm}\left(E_{\mathrm{I}}\right.$ is $2.41 \mathrm{eV}$ ). Consequently, the calculated crystallite size of GQDs is ca. $2.2 \mathrm{~nm}$, a little larger than its average diameter $D_{\mathrm{a}}$ (ca. $2.04 \mathrm{~nm}$ ). This indicates that the basal plane of our GQDs sheet has nearly perfect $s p^{2}$ network. It is clear that the GQDs obtained by annealing GOQDs in argon atmosphere at $1010^{\circ} \mathrm{C}$ are with mostly pristine edges and nearly perfect basal plane. Namely, our GQDs sample is of high quality, which affords one to unveil the intrinsic magnetism of GQDs.

Magnetic properties of the GQDs sample

To investigate the intrinsic magnetism of our GQDs, the magnetic properties were measured by superconducting quantum interference device (SQUID) magnetometer with sensitivity as high as $10^{-8}$ emu. To minimize the measurement error, the sample more than $5 \mathrm{mg}$ was used. Figure $4 \mathrm{a}$ shows the temperature dependence of the mass magnetization $M$ from 2 to $300 \mathrm{~K}$. As found, there is no magnetic ordering signal detected at any temperature. Despite the absence of magnetic ordering, our sample exhibits noticeable paramagnetism at low temperature and diamagnetism at high temperature. After subtracting the linear diamagnetism, the $1 / X-T$ curve was obtained, which fits well with the Curie law $X$ $=C / T$ (Fig. 4b). To characterize the species contributing to the localized magnetic moments, measurements of the mass magnetization $M$ vs magnetic field $H$ at 300 and $2 \mathrm{~K}$ were performed. As noticed from the $M-H$ curve shown in Fig. 4c, the sample shows linear diamagnetism at $300 \mathrm{~K}$. Since the diamagnetic magnetization varies linearly with magnetic field and is independent of temperature, the net mass magnetization $\Delta M$ at $2 \mathrm{~K}$ (Fig. $4 \mathrm{~d}$ ) was obtained by subtracting the diamagnetic magnetization at $300 \mathrm{~K}$. The corresponding $\Delta M-H$ curve of GQDs at $2 \mathrm{~K}$ with error bars given by repeating the measurements is shown in Supplementary Figure S1, indicating the high precision of our magnetic detection. In details, the $\Delta M-H$ curve of GQDs at $2 \mathrm{~K}$ can be described by the standard Brillouin function

$M=M_{\mathrm{S}}\left[\frac{2 J+1}{2 J} \operatorname{Coth}\left(\frac{2 J+1}{2 J} x\right)-\frac{1}{2 J} \operatorname{Coth}\left(\frac{x}{2 J}\right)\right]$,

where $x=g J \mu_{\mathrm{B}} H /\left(k_{\mathrm{B}} T\right)$, the saturated magnetization $M_{\mathrm{s}}=N \mathrm{~g} J \mu_{\mathrm{B}}, g$ is the Lande factor, $k_{\mathrm{B}}$ is the Boltzmann constant, $J$ is the angular momentum number, and $N$ is the number of present spins. The $\Delta M$ value of GQDs at low magnetic field is small and prone to be affected by thermal fluctuations, while the $\Delta M$ value at high magnetic field is more accurate. Then, our Brillouin function fitting is done on the $\Delta M-H$ curve at high magnetic field. Assuming $g=2$, the Brillouin function fitted $J$ and $M_{\mathrm{s}}$ of GQDs are 0.6 and 0.589 emu/g, respectively. One can find that the $J$ value is close to the quantum number $1 / 2$. Here it should be mentioned that after the magnetic measurements, we carefully performed inductively coupled plasma (ICP) spectrometric measurement to exclude the magnetic contribution of $3 \mathrm{~d}$ impurities. The $3 \mathrm{~d}$ impurity elements of the sample are negligible with $\mathrm{Fe}<65 \mathrm{ppm}, \mathrm{Cr}<35 \mathrm{ppm}, \mathrm{Mn}$, 
a

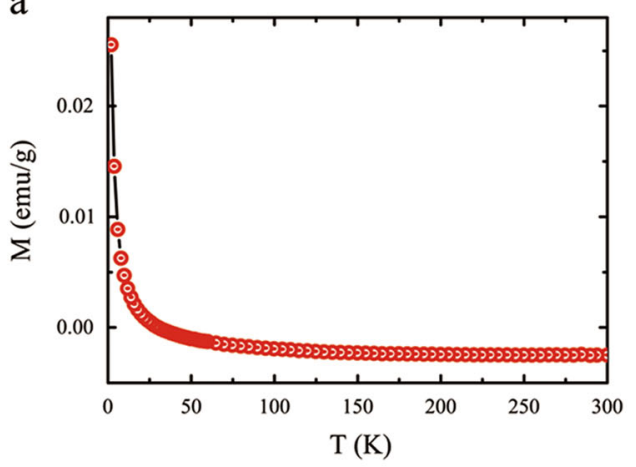

c

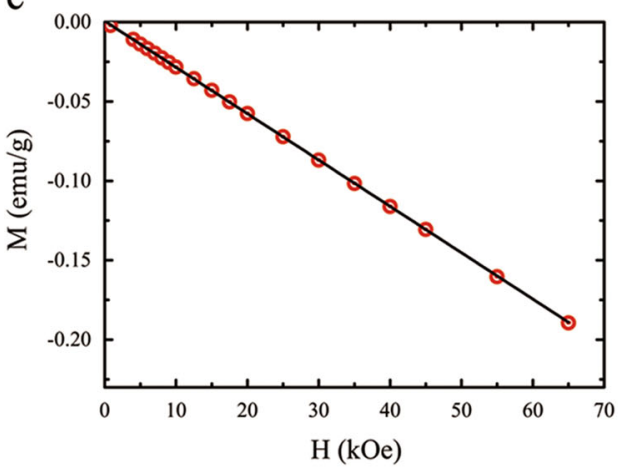

$\mathrm{b}$

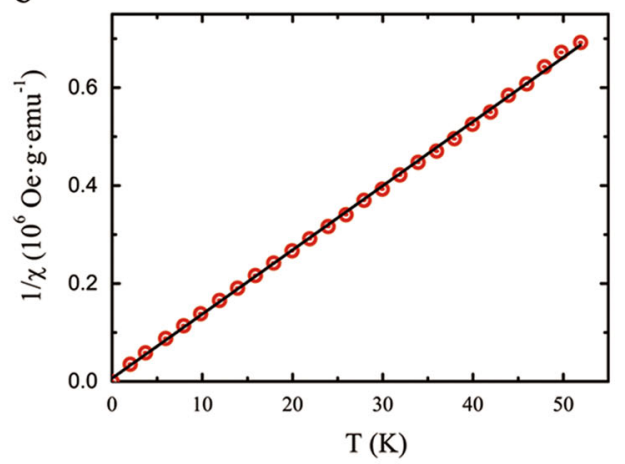

d

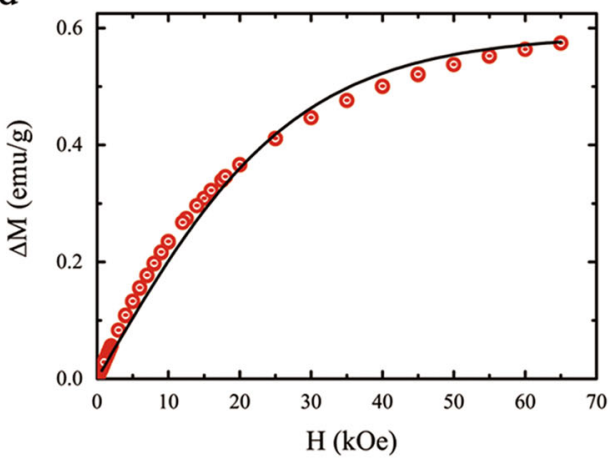

Fig. 4 Magnetic properties of GQDs. a $M-T$ and $\mathbf{b} 1 / X-T$ under the applied field $H=1 \mathrm{kOe}$. The round symbols are the measurements and the solid lines are fitted by the Curie law. c $M-H$ measured at $300 \mathrm{~K}$. d $\Delta M-H$ measured at $2 \mathrm{~K}$. The round symbols are the measurements and the solid lines are fit to Brillouin function. The coefficient of determination for the fitting is 0.9997 indicating the goodness of fitting

$\mathrm{Ni}$, and Co lower than the detection limit. Clearly, one can conclude that the detected magnetism of our GQDs sample is intrinsic.

\section{DISCUSSION}

The magnetic moments of our GQDs sample with high purity are from the intrinsic structure. As reported, the magnetic moments of GO come from two categories: oxygen groups and vacancies on the basal plane, and spin polarized states at zigzag edges. ${ }^{36}$ As mentioned above, the XPS and Raman characterizations indicate that oxygen groups and vacancies on the basal plane have been removed by annealing at the high temperature of $1010^{\circ} \mathrm{C}$. The basal plane of GQDs is nearly perfect, which would not contribute to the magnetism. Thus, the magnetic moments of GQDs may be mainly from the spin polarized edge states. It has been experimentally confirmed that the edges derived by oxidative cutting are mostly well-defined zigzag edges. ${ }^{37,38}$ In addition, the magnetic moment on each carbon atom at the pristine zigzag edge was reported to be $1.2 \mu_{\mathrm{B}}{ }^{39}$ In this case, the magnetic moment of GQDs, in principle, can be arbitrarily large. However, our GQDs with substantial pristine edges obtained by oxidative cutting only exhibited the purely Curie-like paramagnetism even at $2 \mathrm{~K}$ with the magnetic moment of $1.2 \mu_{\mathrm{B}}$. To take a deeper insight into the magnetism of our GQDs, the number of present spins $N$ and the ratio $n$ of magnetic quantum dots to total quantum dots were calculated. The corresponding microstructure and magnetic properties are summarized in Table 1. The number of present spins $N$ was calculated from the fitted $M_{\mathrm{s}}$ and $J$. Then, the ratio $n$ of magnetic quantum dots to total quantum dots was approximately obtained from the average diameter $D_{a}$, the carbon content and the number of present spins $N$. The calculated number of present spins $N$ of our GQDs is $5.28 \times 10^{19} \mathrm{~g}^{-1}$, which is

\begin{tabular}{|c|c|c|c|c|c|c|}
\hline $\begin{array}{l}D_{\mathrm{a}} \\
(\mathrm{nm})\end{array}$ & $\begin{array}{l}L_{\mathrm{a}} \\
(\mathrm{nm})\end{array}$ & $\begin{array}{l}\text { Carbon } \\
\text { content (at.\%) }\end{array}$ & J & $\begin{array}{l}M_{\mathrm{s}} \\
(\mathrm{emu} / \mathrm{g})\end{array}$ & $\begin{array}{l}N \\
(1 / g)\end{array}$ & $n$ \\
\hline 2.04 & 2.2 & 94.58 & 0.6 & 0.589 & $5.28 \times 10^{19}$ & $\sim 1 / 7$ \\
\hline
\end{tabular}

much larger than the values $\left(2.2 \times 10^{18} \mathrm{~g}^{-1}\right)$ reported in the graphene nanocrystals with typical sizes of $10-50 \mathrm{~nm} .{ }^{40}$ Therein, graphene nanocrystals also show paramagnetism, which was reported to be associated with zigzag edges. Since the typical size of our GQDs $(\sim 2.04 \mathrm{~nm})$ is much smaller than that of the graphene nanocrystals, our GQDs have higher edge-to-area ratio and, thus possibly more spin polarized zigzag segments. Despite the high magnetic concentration, the calculated ratio $n$ of magnetic GQDs to total GQDs is approximately $1 / 7$. This implies that the majority of our GQDs are nonmagnetic.

The bare zigzag edges were reported to be inclined, in order to reconstruct into the pentagon-heptagon structures ( $Z Z 57$, shown in Fig. 5a), especially at a high temperature (above $600^{\circ} \mathrm{C}$ ). ${ }^{41,42}$ This reconstructed ZZ57 structure is theoretically considered to be the most stable edge of graphene. ${ }^{43-45}$ Nevertheless, these ZZ57 edges would lead to the loss of the edge states magnetism. ${ }^{39,46,47}$ Since our GQDs were obtained by annealing GOQDs at $1010^{\circ} \mathrm{C}$, the $Z Z 57$ reconstruction at the edge may take place as well, then suppressing the edge states magnetism (as illustrated in Fig. 5a). Moreover, our previous studies of GOQDs have demonstrated that there may be some defects at the edge, which would also suppress the edge states magnetism. ${ }^{25}$ Therefore, both the ZZ57 reconstruction and/or defects at the edges are proposed to be the 
a

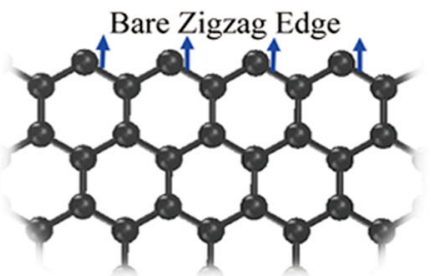

Before Annealing

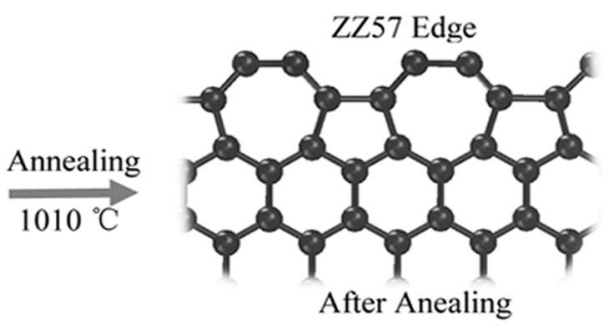

After Anealing

b

Zigzag Edge Passivated by Hydroxyl

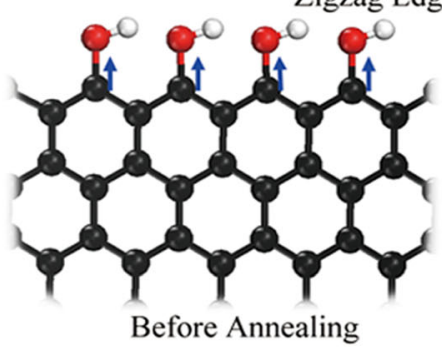

Before Annealing
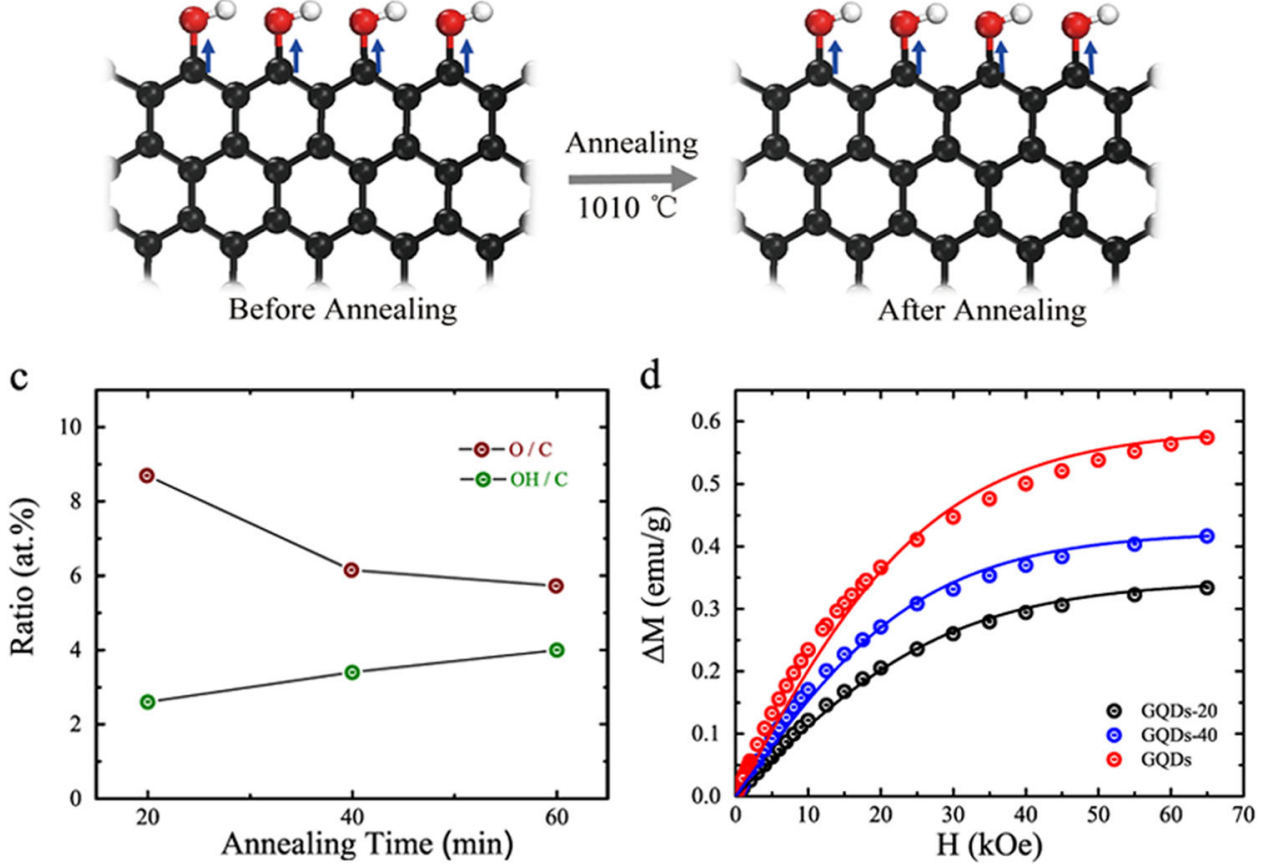

Fig. 5 Schematic illustrations of a bare zigzag edge, $\mathbf{b}$ zigzag edge with $\sigma$ bonds passivated by hydroxyl groups, before and after annealing at $1010^{\circ} \mathrm{C}$. c Dependences of oxygen to carbon ratio $(\mathrm{O} / \mathrm{C}$, wine dots) and $\mathrm{OH}$ coverage $(\mathrm{OH} / \mathrm{C}$, green dots) measured or calculated according to the XPS spectra on the annealing time. The lines shown are guides to the eyes only. d $\Delta M-H$ of GQDs-20 (black dots), GQDs-40 (blue dots) and GQDs (red dots) samples measured at $2 \mathrm{~K}$. The round symbols are the measurements and the solid lines are fit to Brillouin function

reason for the fact that the majority of the quantum dots in our GQDs are nonmagnetic. This indicates that it is difficult to preserve well-defined zigzag edges for the mass-production of GQDs.

Despite the defects and ZZ57 reconstruction at the edges suppressed most of the magnetic edge states, our GQDs still show noticeable paramagnetism at $2 \mathrm{~K}$. From the typical fine-scanned $\mathrm{C}$ 1s spectrum analysis, one can see that some residual oxygen groups are at the edge of GQDs in the form of hydroxyl, ether and carbonyl, which were reported to be thermally stable. ${ }^{27,29}$ Notably, the hydroxyl groups at the edge can survive even at a temperature of $1000{ }^{\circ} \mathrm{C} .{ }^{27}$ It was reported that ether and carbonyl at the edge of graphene are nonmagnetic, ${ }^{36}$ while hydroxyl passivating the $\sigma$ bond of the zigzag edge $C$ can preserve the edge states magnetism. ${ }^{47,48}$ Especially, as reported by Song et al., zigzag edges with passivated $\sigma$ bonds can avoid edge reconstruction. ${ }^{39,47}$ Therefore, there may be some residual zigzag edges with the $\sigma$ bonds passivated by hydroxyl groups, which are free of reconstruction and preserve the edge states magnetism (as illustrated in Fig. 5b). Reasonably, these residual zigzag edges passivated by hydroxyl groups may mainly contribute to the paramagnetism of our GQDs. In addition, the basal-plane defects such as point vacancies or chemisorbed $\mathrm{OH}$ groups on the basal plane of graphene sheet would induce magnetic moments. ${ }^{36,49}$ Despite that our GQDs have nearly perfect basal planes, one can't exclude the possibility that there may still be some residual defects on the basal plane of GQDs sheet. Namely, defects on the basal plane may more or less contribute to the magnetism of our GQDs.

The magnetic correlation length plays the key role in the magnetism of our GQDs. For graphene, the magnetic correlation length was theoretically estimated to be $\sim 100 \mathrm{~nm}$ at $2 \mathrm{~K}^{50}$ two orders of magnitude higher than the size of our GQDs. Thus, the magnetic correlation can be maintained in the whole quantum dot. Considering the fact that both the residual zigzag edges and defects on the basal plane may be the magnetic sources of our GQDs, the edge states magnetism and the basal-plane magnetism would interact with each other to form a magnetic moment. ${ }^{20,50}$ Assuming that all of the hydroxyl groups bond at zigzag edges and contribute to the magnetic moments, the number of present spins $N$ can be calculated from the hydroxyl density. According to the oxygen content and the deconvoluted C 1s spectrum, the density of hydroxyls was approximately obtained to be $\sim 76.28 \times$ $10^{19} \mathrm{~g}^{-1}$. As an unit cell of zigzag edge with passivated $\sigma$ bond was reported to yield a magnetic moment of $0.3 \mu_{\mathrm{B}}{ }^{39,50}$ the magnetic moment of $1.2 \mu_{\mathrm{B}}$, as obtained for our GQDs, which just corresponds to four zigzag-edge unit cells. Then, the number of present spins $N$ was calculated to be $\sim 19.07 \times 10^{19} \mathrm{~g}^{-1}$, which is more than that obtained from the saturated magnetization $M_{s}$ $\left(\sim 5.28 \times 10^{19} \mathrm{~g}^{-1}\right)$. Given the facts that (i) hydroxyl groups may also bond at non-zigzag edges, (ii) the distribution of hydroxyl 
groups is non-uniform, and (iii) antiferromagnetic coupling of the sublattices would induce the spins cancellation, ${ }^{11}$ one understands that the $N$ of our GQDs (obtained from $M_{\mathrm{s}}$ ) is naturally less than that calculated from the hydroxyl groups. Therefore, it further suggests that the hydroxyl passivated zigzag edges contribute to the magnetism of GQDs.

To further investigate the effect of hydroxyl on preserving the zigzag edge states magnetism, we annealed GOQDs at the temperature of $1010^{\circ} \mathrm{C}$ for 20 and $40 \mathrm{~min}$ to obtain the samples GQDs-20 and GQDs-40 with different oxide degrees and contents of hydroxyl. The XPS spectra and the fine-scanned $C 1 \mathrm{~s}$ spectra of GQDs-20, GQDs-40 and GQDs samples are shown in Supplementary Figure $\mathrm{S} 2 \mathrm{a}$ and $\mathrm{b}$. Figure $5 \mathrm{c}$ shows the dependence of $\mathrm{O} / \mathrm{C}$ and $\mathrm{OH} / \mathrm{C}$ on the annealing time. Despite the oxidation degree of the sample decreased, the hydroxyl content of the sample increases with the annealing time. The reason may be that some oxygen groups convert to the hydroxyl groups at the edge during the annealing. ${ }^{27,36}$ The $M-T$ and $1 / X-T$ curves of GQDs-20 and GQDs-40 are shown in Supplementary Figure S3a and b. Both samples show the purely Curie-like paramagnetism as GQDs. The $\Delta M-H$ curves of GQDs-20, GQDs-40 and GQDs at $2 \mathrm{~K}$ are shown in Fig. $5 \mathrm{~d}$, which can be fitted by the standard Brillouin function. The fitted $J$ values are 0.5 for GQDs- 20 and 0.6 for GQDs-40, close to the quantum number $1 / 2$ as GQDs. The calculated numbers of present spins $N$ are $3.72 \times 10^{19} \mathrm{~g}^{-1}$ for GQDs-20, $3.81 \times 10^{19} \mathrm{~g}^{-1}$ for GQDs-40 and $5.28 \times 10^{19} \mathrm{~g}^{-1}$ for GQDs, respectively. Clearly, with the increase of the hydroxyl groups, the spin density of the sample increased. Namely, one can propose that the increased spin density comes from the increased hydroxyl groups. This indicates that the hydroxyl groups at the zigzag edge contribute to the preservation of edge states magnetism, and the magnetic moments of our GQDs come from the hydroxyl groups passivated zigzag edges.

The edge states magnetism is crucial for the application of GQDs in spintronics. Nevertheless, the defects and ZZ57 reconstruction at the edge would suppress most of the edge states magnetism. As mentioned above, zigzag edge with passivated $\sigma$ bond is thermally stable ${ }^{39}$, and can preserve the magnetic edge states. ${ }^{47,48}$ Therefore, GQDs with well-defined magnetic edge states may be obtained by passivating the $\sigma$ bond at the edge of GOQDs with hydroxyl firstly, and then annealing at high temperature to remove the basal-plane oxygen and recover the $s p^{2}$ network structure. This may provide a new guidance to obtain GQDs with magnetic edges.

\section{CONCLUSION}

The GQDs with high-purity and mass production were obtained, which have mostly pristine edges and nearly perfect basal planes. The GQDs show the purely Curie-like paramagnetism with the local moment of $1.2 \mu_{\mathrm{B}}$ at $2 \mathrm{~K}$. The majority of the GQDs are nonmagnetic. Defects and/or reconstruction at the edges arising from high-temperature annealing are proposed to suppress most of the edge-states magnetism. The residual zigzag edges passivated by hydroxyl groups are proposed to be the mainly magnetic sources of GQDs. Our work provides the important insights into the intrinsic magnetism of GQDs. Furthermore, passivating the zigzag edge by hydroxyl can be a promising method to obtain GQDs with magnetic edges, which may be crucial for applications in spintronics.

\section{MATERIALS AND METHODS}

Sample synthesis

Monolayer GOQDs as the precursor with high yield and purity were produced by acid-assisted cleavage of carbon black as reported previously. ${ }^{25}$ The obtained GOQDs were put into a graphite boat, then flushed with argon in a quartz tube furnace for $30 \mathrm{~min}$ to discharge the air. Then the samples were heated to $1010^{\circ} \mathrm{C}$, and then maintained for $20 \mathrm{~min}$ (GQDs-20), $40 \mathrm{~min}$ (GQDs-40) and $60 \mathrm{~min}$ (GQDs) flushed with argon, respectively. After that, the samples were naturally cooled to temperature in argon atmosphere to generate the final GQDs-20, GQDs-40 and GQDs60 samples.

\section{Sample characterization}

The morphology of GQDs was investigated by TEM (JEM-2100, Japan) and AFM (SPI-3800N, Japan). The XPS measurement was performed on PHI5000 VersaProbe using Al Ka radiation. The Raman spectra were obtained by an InVia Raman system (Renishaw, England) using $514 \mathrm{~nm}$ laser as the light source.

\section{Magnetic measurements}

SQUID magnetometer with sensitivity better than $10^{-8}$ emu (MPMS-XL, USA) was used to examine the magnetic properties of the GQDs. To verify the $3 d$ impurity elements of the sample, we carefully performed ICP spectrometric measurements (Jarrell-Ash, USA).

\section{ACKNOWLEDGEMENTS}

This work was financially supported by the State Key Program for Basic Research (Grant No. 2014CB921102), and NSFC (Grant No. 51572122), China. A portion of this work was performed on the Steady High Magnetic Field Facilities, High Magnetic Field Laboratory, Chinese Academy of Sciences.

\section{AUTHOR CONTRIBUTIONS}

N.J.T. conceived the project and designed the experiments with Y.Y.S. Y.Y.S. contributed to sample preparation. Y.Y.S. carried out magnetic measurements. Y.Y.S and N.J.T. analyzed the data. N.J.T. and Y.Y.S. wrote the manuscript. All authors discussed the results and commented on the manuscript.

\section{COMPETING INTERESTS}

The authors declare no competing interests.

\section{REFERENCES}

1. Ponomarenko, L. A. et al. Chaotic dirac billiard in graphene quantum dots. Science 320, 356-358 (2008).

2. Ritter, K. A. \& Lyding, J. W. The influence of edge structure on the electronic properties of graphene quantum dots and nanoribbons. Nat. Mater. 8, 235-242 (2009).

3. Wang, X., Sun, G., Li, N. \& Chen, P. Quantum dots derived from two-dimensional materials and their applications for catalysis and energy. Chem. Soc. Rev. 45, 2239-2262 (2016).

4. Wang, W. L., Yazyev, O. V., Meng, S. \& Kaxiras, E. Topological frustration in graphene nanoflakes: magnetic order and spin logic devices. Phy. Rev. Lett 102, 157201 (2009).

5. Li, L. S. \& Yan, X. Colloidal graphene quantum dots. J. Phys. Chem. Lett. 1, 2572-2576 (2010).

6. Enoki, T. T. \& Takai, K. Unconventional electronic and magnetic functions of nanographene-based host-guest systems. Dalton Trans. 29, 3773-3781 (2008).

7. Wang, W. L., Meng, S. \& Kaxiras, E. Graphene nanoflakes with large spin. Nano Lett. 8, 241-245 (2008).

8. Şahin, H., Senger, R. T. \& Ciraci, S. Spintronic properties of zigzag-edged triangular graphene flakes. J. Appl. Phys. 108, 074301 (2010).

9. Fernández-Rossier, J. \& Palacios, J. J. Magnetism in graphene nanoislands. Phys. Rev. Lett. 99, 177204 (2007).

10. Ezawa, M. Metallic graphene nanodisks: electronic and magnetic properties. Phys. Rev. B 76, 245415 (2007).

11. Espinosa-Ortega, T., Luk'yanchuk, I. A. \& Rubo, Y. G. Magnetic properties of graphene quantum dots. Phys. Rev. B 87, 205434 (2013).

12. Yazyev, O. V. Emergence of magnetism in graphene materials and nanostructures. Rep. Prog. Phys. 73, 056501 (2010).

13. Kabir, M. \& Saha-Dasgupta, T. Manipulation of edge magnetism in hexagonal graphene nanoflakes. Phys. Rev. B 90, 035403 (2014).

14. Magda, G. Z. et al. Room-temperature magnetic order on zigzag edges of narrow graphene nanoribbons. Nature 514, 608-611 (2014). 
15. Heiskanen, H. P., Manninen, M. \& Akola, J. Electronic structure of triangular, hexagonal and round graphene flakes near the Fermi level. New. J. Phys. 10, 103015 (2008).

16. Jiang, J., Lu, W. \& Bernholc, J. Edge states and optical transition energies in carbon nanoribbons. Phys. Rev. Lett. 101, 246803 (2008).

17. Huang, B., Liu, F., Wu, J., Gu, B. L. \& Duan, W. Suppression of spin polarization in graphene nanoribbons by edge defects and impurities. Phys. Rev. B 77, 153411 (2008).

18. Kunstmann, J., Özdoğan, C., Quandt, A. \& Fehske, H. Stability of edge states and edge magnetism in graphene nanoribbons. Phys. Rev. B 83, 045414 (2011).

19. Akola, J., Heiskanen, H. \& Manninen, M. Edge-dependent selection rules in magic triangular graphene flakes. Phys. Rev. B 77, 193410 (2008).

20. Wimmer, M., Akhmerov, A. R. \& Guinea, F. Robustness of edge states in graphene quantum dots. Phys. Rev. B 82, 045409 (2010).

21. Espinosa-Ortega, T., Luk'yanchuk, I. A. \& Rubo, Y. G. Density of states in randomly shaped graphene quantum dots. Superlattices Microstruct. 49, 283-287 (2011).

22. Bhowmick, S. \& Shenoy, V. B. Edge state magnetism of single layer graphene nanostructures. J. Chem. Phys. 128, 244717 (2008).

23. Jaworowski, B., Potasz, P. \& Wojs, A. Disorder induced loss of magnetization in Lieb's graphene quantum dots. Superlattices Microstruct 64, 44-51 (2013).

24. Li, L. et al. Focusing on luminescent graphene quantum dots: current status and future perspectives. Nanoscale 5, 4015-4039 (2013).

25. Sun, Y. Y. et al. Intrinsic magnetism of monolayer graphene oxide quantum dots. Appl. Phys. Lett. 108, 033105 (2016).

26. Hontoria-Lucas, C., López-Peinado, A. J., López-González, J. de D., Rojas-Cervantes, M. L. \& Martín-Aranda, R. M. Study of oxygen-containing groups in a series of graphite oxides: physical and chemical characterization. Carbon $N$ Y 33, 1585-1592 (1995).

27. Ganguly, A., Sharma, S., Papakonstantinou, P. \& Hamilton, J. Probing the thermal deoxygenation of graphene oxide using high-resolution in situ X-ray-based spectroscopies. J. Phys. Chem. C 115, 17009-17019 (2011).

28. Gao, X., Jang, J. \& Nagase, S. Hydrazine and thermal reduction of graphene oxide: reaction mechanisms, product structures, and reaction design. J. Phys. Chem. C 114, 832-842 (2010).

29. Acik, M. et al. The role of oxygen during thermal reduction of graphene oxide studied by infrared absorption spectroscopy. J. Phys. Chem. C 115, 19761-19781 (2011).

30. Acik, M. et al. Reconstructed ribbon edges in thermally reduced graphene nanoribbons. J. Phys. Chem. C 116, 24006-24015 (2012).

31. Mattevi, C. et al. Evolution of electrical, chemical, and structural properties of transparent and conducting chemically derived graphene thin films. Adv. Funct. Mater. 19, 2577-2583 (2009).

32. Tuinstra, F. \& Koenig, J. L. Raman spectrum of graphite. J. Chem. Phys. 53 1126-1130 (1970).

33. Diane, S. K. \& William, B. W. Characterization of diamond films by Raman spec troscopy. J. Mater. Res. 4, 385-393 (1989).

34. Cançado, L. G. et al. General equation for the determination of the crystallite size La of nanographite by Raman spectroscopy. Appl. Phys. Lett. 88, 163106 (2006).
35. Mallet-Ladeira, P. et al. A Raman study to obtain crystallite size of carbon materials: a better alternative to the Tuinstra-Koenig law. Carbon N Y 80, 629-639 (2014).

36. Tang, T. et al. Robust magnetic moments on the basal plane of the graphene sheet effectively induced by $\mathrm{OH}$ groups. Sci. Rep. 5, 8448 (2015).

37. Fujii, S. \& Enoki, T. Cutting of oxidized graphene into nanosized pieces. J. Am. Chem. Soc. 132, 10034-10041 (2010).

38. Li, Z. Y. et al. How graphene is cut upon oxidation? J. Am. Chem. Soc. 131, 6320-6321 (2009).

39. Song, L. L., Zheng, X. H., Wang, R. L. \& Zeng, Z. Dangling bond states, edge magnetism, and edge reconstruction in pristine and $\mathrm{B} / \mathrm{N}$-terminated zigzag graphene nanoribbons. J. Phys. Chem. C 114, 12145-12150 (2010).

40. Sepioni, M. et al. Limits on intrinsic magnetism in graphene. Phys. Rev. Lett. 105 207205 (2010).

41. He, K. et al. Temperature dependence of the reconstruction of zigzag edges in graphene. ACS Nano 9, 4786-4795 (2015).

42. Zhang, Z., Kutana, A. \& Yakobson, B. I. Edge reconstruction-mediated graphene fracture. Nanoscale 7, 2716-2722 (2015).

43. Koskinen, P., Malola, S. \& Häkkinen, H. Self-passivating edge reconstructions of graphene. Phys. Rev. Lett. 101, 115502 (2008).

44. Koskinen, P., Malola, S. \& Häkkinen, H. Evidence for graphene edges beyond zigzag and armchair. Phys. Rev. B 80, 073401 (2009).

45. Gan, C. K. \& Srolovitz, D. J. First-principles study of graphene edge properties and flake shapes. Phys. Rev. B 81, 125445 (2010).

46. Dutta, S. \& Pati, S. K. Edge reconstructions induce magnetic and metallic behavior in zigzag graphene nanoribbons. Carbon N Y 48, 4409-4413 (2010).

47. Voznyy, O., Güçlü, A. D., Potasz, P. \& Hawrylak, P. Effect of edge reconstruction and passivation on zero-energy states and magnetism in triangular graphene quantum dots with zigzag edges. Phys. Rev. B 83, 165417 (2011).

48. Zhao, M., Yang, F., Xue, Y., Xiao, D. \& Guo, Y. Effects of edge oxidation on the stability and half-metallicity of graphene quantum dots. ChemPhysChem 15 157-164 (2014).

49. Yazyev, O. V. Magnetism in disordered graphene and irradiated graphite. Phys. Rev. Lett. 101, 037203 (2008).

50. Yazyev, O. \& Katsnelson, M. I. Magnetic correlations at graphene edges: basis for novel spintronics devices. Phys. Rev. Lett. 100, 047209 (2008).

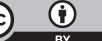

This work is licensed under a Creative Commons Attribution 4.0 International License. The images or other third party material in this article are included in the article's Creative Commons license, unless indicated otherwise in the credit line; if the material is not included under the Creative Commons license, users will need to obtain permission from the license holder to reproduce the material. To view a copy of this license, visit http://creativecommons.org/licenses/by/ 4.0/

(c) The Author(s) 2017

Supplementary Information accompanies the paper on the npj Quantum Materials website (doi:10.1038/s41535-017-0010-2). 